\title{
Biomimetic Synthesis of Ceramic Composites
}

\author{
Pouya Moghimian ${ }^{1}, \underline{\text { Vesna Srot }^{1}}$, Sandra J. Facey ${ }^{2}$ and Peter A. van Aken ${ }^{1}$ \\ 1. Stuttgart Center for Electron Microscopy, Max Planck Institute for Solid State Research, Stuttgart, \\ Germany. \\ ${ }^{2 .}$ Institute of Technical Biochemistry, University of Stuttgart, Stuttgart, Germany.
}

Biomimetic synthesis of ceramics at moderate temperatures by molecular precursors such as proteins or viruses allows for the incorporation of organic component into an inorganic network (e.g. ceramics) which leads to hybrid organic-inorganic composites. This capability of biomolecules of directing the assembly and forming unique structures has raised enormous interest into developing pathways to mimic the nucleation capabilities of biological systems via interfacial interactions (e.g. hydrogen bonding or electrostatic interactions). The synthesis of materials that resemble complex morphologies of natural biominerals is one of the key fields in biomimetic science. M13 phages (Ff class of phages) have been in wide use for a variety of applications in biotechnology as biomimetic matrix for tissue engineering and also as biological templates for material design. Their elongated shape, monodispersity and capability of self-assembling into liquid crystal structures provide materials scientists with modifiable surfaces for the fabrication of hybrid structures. The M13 phage is a filamentous virus with an high aspect ratio L/D $\approx$ 130 , where $\mathrm{L}$ is its contour length $(\sim 880 \mathrm{~nm})$ and $\mathrm{D}$ is its diameter $(\sim 6.6 \mathrm{~nm})$ and a molecular weight of $12 \times 10^{6} \mathrm{Da}$. They are composed of 5 different coat proteins: The P8 coat proteins ( 2700 copies) form a $\sim 2 \mathrm{~nm}$ thick shell around the single stranded DNA (ssDNA). Spontaneous assembly of such colloidal particles can be achieved by evaporation of the phage-containing solution on a substrate. Owing to these features, phages are great candidates for the development of novel bionanomaterials [1-4].

Our first aim in this study is to find conditions in which phage assemblies can keep their primary structure integrity upon exposure to organic solvents. This can be used in the developing topic of research that is the control of macromolecular assemblies with hierarchical frameworks. Here we show that M13 phages would be denaturalized in polar organic solvents as compared to nonpolar solvents. The exposure of M13 phages to apolar solvents (e.g. hexane) did not denature the structure of the phages for up to $8 \mathrm{~h}$. In contrast, phages showed decomposition into rod-like I-forms and to flattened spheroids upon exposure to polar organic solvents. Hexane, that is a non-polar hydrophobic organic solvent, was shown to be incapable to compete for the hydrogen bonds between the phage coat proteins and therefore it was not able to deform the native structure of the phages (Fig. 1a). In contrast, polar solvents used in our study (chloroform, tetrahydrofuran and toluene) were able to compete for the hydrophobic interactions and hydrogen bonding between the phage coat proteins and finally caused the viral structure to collapse (Fig. 1b-d). It was concluded that the fragmentation and as a consequence denaturation of phages can be understood by considering three factors: solvent hydrophobicity, hydrogen-binding capacity and water miscibility [2].

We employed M13 phages as a soft and elastic scaffold for the layer-by-layer construction of phage$\mathrm{ZnO}$ (zinc oxide) structures where the ceramic material is templated by the organic component. This is of high interest due to the broad range of applications of these structures. Here, we used our in-housebuilt convective assembly apparatus to assemble densely-packed and directionally oriented wild-type M13 phage layers on C-coated Si wafers. Because, a-C surface showed to be a promising host for aligned phages [3]. As shown in Figure 2, very thin alternating $\mathrm{ZnO}$ layers of about $20 \mathrm{~nm}$ was grown 
on assembled phage layers using a $\mathrm{ZnO}$ mineralization solution. The microstructure, elemental composition, and also the integrity of the alternating biologically-templated inorganic structures were characterized using scanning electron microscopy (SEM), (scanning) transmission electron microscopy ((S)TEM) and atomic force microscopy (AFM). A homogeneous organic-inorganic multilayered structure without significant thickness fluctuations and with limited interpenetration of the interfaces was achieved. The root mean squared (RMS) roughness of the resulting multilayered structure was $\sim 14$ $\mathrm{nm}$ which is only double of a single phage thickness. Our work can set the stage for templating of organic-inorganic multilayers at the nanoscale, which might have potential in the construction of novel multicomponents (e.g. nacre-inspired structures) with controlled organic to inorganic ratios [4, 5].

\section{References:}

[1] R. Naik et al, Journal of nanoscience and nanotechnology, 2 (2002), p. 95-100.

[2] P. Moghimian et al, International Journal of Materials Research (IJMR), 107 (2016), p. 295-299.

[3] P. Moghimian et al, Langmuir, 30 (2014), p. 11428-11432.

[4] P. Moghimian et al, Journal of Biomaterials and Nanobiotechnology, 7 (2016), p. 72-77.

[5] The authors acknowledge funding from the German Research Foundation (DFG).

\section{Hexane}

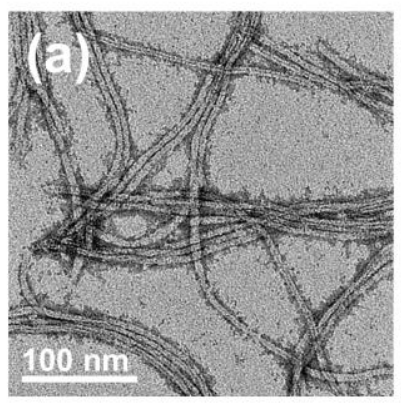

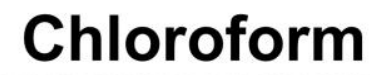

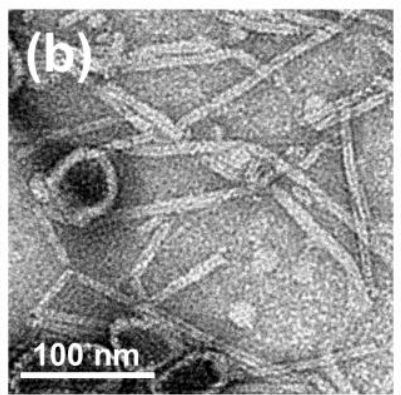

THF

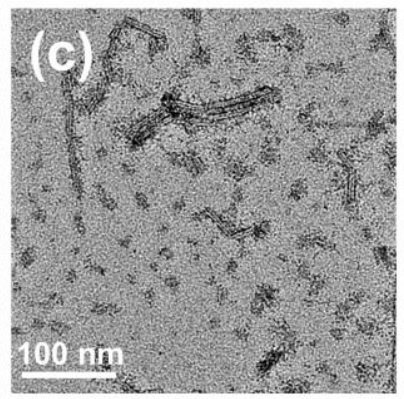

Toluene

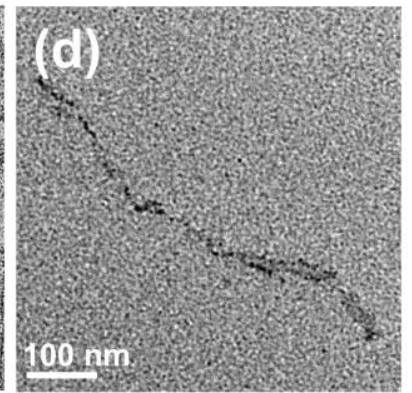

Figure 1. Bright-field TEM (BF-TEM) images of M13 phages and their structural changes after $1 \mathrm{~h}$ exposure to (a) hexane, (b) chloroform, (c) tetrahydrofuran (THF) and (d) toluene.
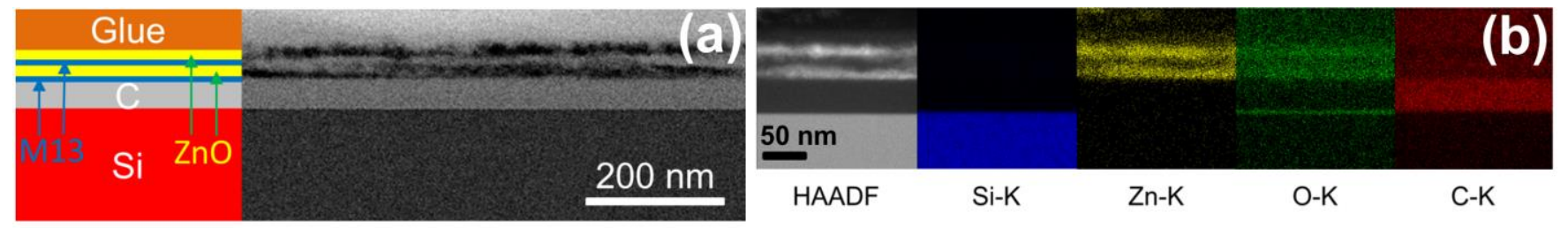

Figure 2. (a) Bright-field STEM (BF-STEM) image of the cross-sectional layered sample, indicating two homogeneous and thin layers of M13 phage and ZnO assembled on a C-sputtered Si substrate. (b) STEM energy-dispersive X-ray spectroscopy (STEM-EDX) elemental mapping derived from the corresponding high-angle annular dark-field (HAADF) image. 